\title{
АСИМПТОТИКА РЕШЕНИЯ СИНГУЛЯРНО ВОЗМУЩЕННОЙ ЗАДАЧИ БЫСТРОДЕЙСТВИЯ С ДВУМЯ МАЛЫМИ ПАРАМЕТРАМИ
}

\author{
А. Р. Данилин, О. О. Коврижных ${ }^{1}$
}

Настоящая работа является продолжением исследования авторов и посвящена задаче оптимального быстродействия для сингулярно возмущенной линейной автономной системы с двумя независимыми малыми параметрами и гладкими геометрическими ограничениями на управление в виде шара

$$
\begin{cases}\dot{x}=y, & x, y \in \mathbb{R}^{2}, \quad u \in \mathbb{R}^{2}, \\ \varepsilon^{3} \dot{y}=J y+u, & \|u\| \leqslant 1, \quad 0<\varepsilon, \mu \ll 1, \\ x(0)=x_{0}(\varepsilon, \mu)=\left(x_{0,1}, \varepsilon^{3} \mu \xi\right)^{*}, \quad y(0)=y_{0}, & \\ x\left(T_{\varepsilon, \mu}\right)=0, \quad y\left(T_{\varepsilon, \mu}\right)=0, \quad T_{\varepsilon, \mu} \longrightarrow \min , & \end{cases}
$$

где

$$
J=\left(\begin{array}{ll}
0 & 1 \\
0 & 0
\end{array}\right) .
$$

Основное отличие от ранее рассмотренных систем с быстрыми и медленными переменными заключается в том, что в данном случае матрица $J$ при быстрых переменных представляет собой жорданову клетку второго порядка с нулевым собственным числом и тем самым не удовлетворяет стандартному условию асимптотической устойчивости. Кроме того, рассмотрены начальные условия, зависящие от второго малого параметра $\mu$. Получена и обоснована полная асимптотика времени быстродействия и оптимального управления в смысле Эрдейи по асимптотической последовательности $\varepsilon^{\gamma}\left(\varepsilon^{k}+\mu^{k}\right), 0<\gamma<1$.

Ключевые слова: оптимальное управление, задача быстродействия, асимптотическое разложение, сингулярно возмущенная задача, малый параметр.

A. R. Danilin, O. O. Kovrizhnykh. Asymptotics of the solution to a singularly perturbed timeoptimal control problem with two small parameters.

The paper continues the author's previous studies. We consider a time-optimal control problem for a singularly perturbed linear autonomous system with two independent small parameters and smooth geometric constraints on the control in the form of a ball

$$
\begin{cases}\dot{x}=y, & x, y \in \mathbb{R}^{2}, \quad u \in \mathbb{R}^{2}, \\ \varepsilon^{3} \dot{y}=J y+u, & \|u\| \leqslant 1, \quad 0<\varepsilon, \mu \ll 1, \\ x(0)=x_{0}(\varepsilon, \mu)=\left(x_{0,1}, \varepsilon^{3} \mu \xi\right)^{*}, \quad y(0)=y_{0}, & \\ x\left(T_{\varepsilon, \mu}\right)=0, \quad y\left(T_{\varepsilon, \mu}\right)=0, \quad T_{\varepsilon, \mu} \longrightarrow \min , & \end{cases}
$$

where

$$
J=\left(\begin{array}{ll}
0 & 1 \\
0 & 0
\end{array}\right) .
$$

The main difference of this case from the systems with fast and slow variables studied earlier is that here the matrix $J$ at the fast variables is the second-order Jordan block with zero eigenvalue and, thus, does not satisfy the standard asymptotic stability condition. Continuing the research, we consider initial conditions depending on the second small parameter $\mu$. We derive and justify a complete asymptotic expansion in the sense of Erdelyi of the optimal time and optimal control with respect to the asymptotic sequence $\varepsilon^{\gamma}\left(\varepsilon^{k}+\mu^{k}\right), 0<\gamma<1$.

Keywords: optimal control, time-optimal control problem, asymptotic expansion, singularly perturbed problem, small parameter.

MSC: 93C70, 49N05

DOI: $10.21538 / 0134-4889-2019-25-2-88-101$

\footnotetext{
${ }^{1}$ Работа выполнена при частичной поддержке Программы повышения конкурентоспособности ведущих университетов РФ (Соглашение с Минобрнауки РФ 02.А03.21.0006 от 27 августа 2013 г.).
} 


\section{1. Постановка задачи}

В работе рассматривается одна из задач о быстродействии оптимального управления [1] для линейной автономной системы с быстрыми и медленными переменными (см. обзор [2]) в классе кусочно-непрерывных управлений с гладкими геометрическими ограничениями

$$
\begin{cases}\dot{x}=y, & x, y \in \mathbb{R}^{2}, \quad u \in \mathbb{R}^{2}, \\ \varepsilon^{3} \dot{y}=J y+u, & \|u\| \leqslant 1, \quad 0<\varepsilon, \mu \ll 1, \\ x(0)=x_{0}(\varepsilon, \mu)=\left(x_{0,1}, \varepsilon^{3} \mu \xi\right)^{*}, \quad y(0)=y_{0}, & \\ x\left(T_{\varepsilon, \mu}\right)=0, \quad y\left(T_{\varepsilon, \mu}\right)=0, \quad T_{\varepsilon, \mu} \longrightarrow \min , & \end{cases}
$$

где $\xi \in \mathbb{R}$ - известная константа, $\xi \neq 0$; * обозначает операцию транспонирования;

$$
J=\left(\begin{array}{cc}
0 & 1 \\
0 & 0
\end{array}\right)
$$

Отметим, что малый параметр $\varepsilon$ входит в уравнения системы (1.1) в третьей степени для удобства, чтобы избежать в дальнейшем дробных рациональных степеней параметра в асимптотиках.

Ранее в работе [3] были получены основные соотношения для системы общего вида с многоугольником в качестве ограничивающего множества, в работах $[4 ; 5]$ исследовано поведение областей достижимости при стремлении малого параметра к нулю. Статьи [6-8] посвящены получению полной асимптотики решения в задачах управления для линейных систем с быстрыми и медленными переменными и ограничивающим множеством в виде шара в евклидовом пространстве. Отличительная особенность рассматриваемой задачи заключается в том, что собственные значения матрицы при быстрых переменных равны нулю и тем самым нарушено стандартное условие (см., например, [5, гл. 3, п. 3.2, предположение А1]) асимптотической устойчивости этой матрицы. В настоящей работе используются методы статьи [6] и общие соотношения, полученные в [9]. Отметим статью [10], в ней впервые была исследована асимптотика решения для другой системы, в которой матрица при быстрых переменных не удовлетворяет условию асимптотической устойчивости.

Настоящая работа является продолжением исследования авторов, опубликованного в 2018 г. (Тр. Ин-та математики и механики УрО РАН, Т. 24, № 2, С. 76-92), в котором в многомерном случае доказана разрешимость задачи, зависящей от двух малых параметров, получена асимптотика решения при $\mu=0$ относительно одного малого параметра $\varepsilon$.

На указанную статью будем далее ссылаться как на предшествующую. Цель настоящего исследования - получить асимптотические разложения времени быстродействия и оптимального управления в рассматриваемой задаче относительно двух независимых малых параметров $\varepsilon$ и $\mu$. Несмотря на то, что задача разрешима при любых начальных данных, будут введены условия общего положения на некоторые координаты начального вектора. При невыполнении этих условий исследование асимптотики решения данной задачи значительно усложнится.

\section{2. Основная система уравнений}

Обозначим

$$
\begin{gathered}
\mathcal{A}_{\varepsilon}=\left(\begin{array}{cc}
0 & I \\
0 & \varepsilon^{-3} J
\end{array}\right), \quad \mathcal{B}_{\varepsilon}=\left(\begin{array}{c}
0 \\
\varepsilon^{-3} I
\end{array}\right), \quad z=\left(\begin{array}{c}
x \\
y
\end{array}\right), \quad z_{0}(\varepsilon, \mu)=\left(\begin{array}{c}
x_{0}(\varepsilon, \mu) \\
y_{0}
\end{array}\right), \\
x=\left(\begin{array}{c}
x_{1} \\
x_{2}
\end{array}\right), \quad y=\left(\begin{array}{c}
y_{1} \\
y_{2}
\end{array}\right), \quad u=\left(\begin{array}{l}
u_{1} \\
u_{2}
\end{array}\right), \quad x_{i}, y_{i}, u_{i} \in \mathbb{R}, \quad i=1,2 .
\end{gathered}
$$


Здесь и далее $I$ - матрица тождественного отображения $\mathbb{R}^{2} \rightarrow \mathbb{R}^{2}$. Вследствие критерия Калмана (см., например, [11, с. 91, теорема 5]) при каждом фиксированном $\varepsilon>0$ система (1.1) с матрицами $\left(\mathcal{A}_{\varepsilon}, \mathcal{B}_{\varepsilon}\right)$ из $(2.1)$ вполне управляема.

В силу принципа максимума Понтрягина [1; 11, гл. 2, теорема 18], который в рассматриваемом случае является необходимым и достаточным условием оптимальности, существует такой вектор $r_{\varepsilon, \mu} \in \mathbb{R}^{4}$, что оптимальное управление в задаче (1.1) имеет вид

$$
u_{\varepsilon, \mu}(t)=\frac{\mathcal{B}_{\varepsilon}^{*} e^{-\mathcal{A}_{\varepsilon}^{*} t} r_{\varepsilon, \mu}}{\left\|\mathcal{B}_{\varepsilon}^{*} e^{-\mathcal{A}_{\varepsilon}^{*} t} r_{\varepsilon, \mu}\right\|}
$$

при всех таких $t$, что $\mathcal{B}_{\varepsilon}^{*} e^{-\mathcal{A}_{\varepsilon}^{*} t} r_{\varepsilon, \mu} \neq 0$. Тогда в силу формулы Коши из $(1.1)$ для $r_{\varepsilon, \mu}$ получим векторное равенство

$$
0=z_{0}(\varepsilon, \mu)+\int_{0}^{T_{\varepsilon, \mu}} \frac{e^{-\mathcal{A}_{\varepsilon} t} \mathcal{B}_{\varepsilon} \mathcal{B}_{\varepsilon}^{*} e^{-\mathcal{A}_{\varepsilon}^{*} t} r_{\varepsilon, \mu}}{\left\|\mathcal{B}_{\varepsilon}^{*} e^{-\mathcal{A}_{\varepsilon}^{*} t} r_{\varepsilon, \mu}\right\|} d t
$$

Тем самым вектор $r_{\varepsilon, \mu}$ является вектором, порождающим оптимальное управление, тогда и только тогда, когда $r_{\varepsilon, \mu}$ удовлетворяет соотношению (2.3). Таким образом, задача сводится к исследованию уравнения (2.3). Непосредственным вычислением из (1.2), (2.1) и равенства $J^{2}=0$ получаем, что

$$
e^{J t / \varepsilon^{3}}=I+\frac{t}{\varepsilon^{3}} J, \quad e^{\mathcal{A}_{\varepsilon} t}=\left(\begin{array}{cc}
I & t I+\frac{t^{2}}{2 \varepsilon^{3}} J \\
0 & I+\frac{t}{\varepsilon^{3}} J
\end{array}\right)
$$

С учетом (2.4), как и в предыдущей работе, удобно вместо вектора $r_{\varepsilon, \mu}=\left\{r_{i}(\varepsilon, \mu)\right\}, i=\overline{1,4}$, где $r_{i}(\varepsilon, \mu) \in \mathbb{R}$, ввести в рассмотрение новый неизвестный вектор $\bar{r}_{\varepsilon, \mu}$ (опустив указание на зависимость компонент от параметров $\varepsilon$ и $\mu)$, компоненты $\bar{r}_{i}(\varepsilon, \mu)$ которого связаны с $r_{i}(\varepsilon, \mu)$ по формулам

$$
2 \varepsilon^{3} T_{\varepsilon, \mu}^{-1} \bar{r}_{1}=r_{1}, \quad \bar{r}_{2}=r_{2}+\varepsilon^{-3} r_{3}, \quad r_{3}=T_{\varepsilon, \mu} \bar{r}_{3}, \quad r_{4}=T_{\varepsilon, \mu} \bar{r}_{4}
$$

Запишем основную систему уравнений (2.3), сделав замену переменной интегрирования в правой части по формуле $t=T_{\varepsilon, \mu} \tau$,

$$
-z_{0}(\varepsilon, \mu)=T_{\varepsilon, \mu} \int_{0}^{1} e^{-\mathcal{A}_{\varepsilon} T_{\varepsilon, \mu} \tau} \mathcal{B}_{\varepsilon} \Psi_{\bar{r}_{\varepsilon, \mu}}(\tau) d \tau
$$

где в новых обозначениях

$$
\Psi_{\bar{r}_{\varepsilon, \mu}}(\tau)=\frac{\left(\begin{array}{c}
-2 \varepsilon^{3} T_{\varepsilon, \mu}^{-1} \tau \bar{r}_{1}+\bar{r}_{3} \\
\tau^{2} \bar{r}_{1}-\tau \bar{r}_{2}+\bar{r}_{4}
\end{array}\right)}{\left(\left(-2 \varepsilon^{3} T_{\varepsilon, \mu}^{-1} \tau \bar{r}_{1}+\bar{r}_{3}\right)^{2}+\left(\tau^{2} \bar{r}_{1}-\tau \bar{r}_{2}+\bar{r}_{4}\right)^{2}\right)^{1 / 2}} .
$$


Система (2.6) эквивалентна системе

$$
\begin{aligned}
-\varepsilon^{6} x_{0,1} & =-\varepsilon^{3} T_{\varepsilon, \mu}^{2} \int_{0}^{1}(\tau, 0) \Psi_{\bar{r}_{\varepsilon, \mu}}(\tau) d \tau+\frac{1}{2} T_{\varepsilon, \mu}^{3} \int_{0}^{1}\left(0, \tau^{2}\right) \Psi_{\bar{r}_{\varepsilon, \mu}}(\tau) d \tau \\
\varepsilon^{6} \mu \xi & =T_{\varepsilon, \mu}^{2} \int_{0}^{1}(0, \tau) \Psi_{\bar{r}_{\varepsilon, \mu}}(\tau) d \tau \\
\varepsilon^{3}\left(\mu \xi-y_{0,1}\right) & =T_{\varepsilon, \mu} \int_{0}^{1}(1,0) \Psi_{\bar{r}_{\varepsilon, \mu}}(\tau) d \tau \\
-\varepsilon^{3} y_{0,2} & =T_{\varepsilon, \mu} \int_{0}^{1}(0,1) \Psi_{\bar{r}_{\varepsilon, \mu}}(\tau) d \tau .
\end{aligned}
$$

Поскольку выражение (2.7) положительно однородно относительно вектора $\bar{r}_{\varepsilon, \mu}$, то будем считать, что

$$
\left\|\bar{r}_{\varepsilon, \mu}\right\|=1
$$

В силу (2.9) множество векторов $\bar{r}_{\varepsilon, \mu}$ ограничено и, следовательно, имеет предельные точки. Пусть $\bar{r}_{0}-$ одна из них, т. е. некоторая последовательность $\bar{r}_{\varepsilon_{n}, \mu_{n}} \rightarrow \bar{r}_{0}$ при $\left(\varepsilon_{n}, \mu_{n}\right) \underset{n \rightarrow \infty}{\longrightarrow}(0,0)$. Опустим далее в рассуждениях индекс $n$ у членов последовательностей $\left(\varepsilon_{n}, \mu_{n}\right)$.

Из рассуждений, аналогичных приведенным в предшествующей работе авторов, следует, что при выполнении условий

$$
x_{0,1} \neq 0, \quad y_{0,1} \neq 0
$$

уравнения (2.8a), (2.8c), (2.8d) влекут справедливость следующих соотношений

$$
\begin{gathered}
\vartheta_{\varepsilon, \mu}:=\frac{\varepsilon^{3}}{T_{\varepsilon, \mu}}=o(1), \quad \varepsilon, \mu \rightarrow 0, \\
\int_{0}^{1} \Psi_{\bar{r}_{0}}(\tau) d \tau=0 .
\end{gathered}
$$

В силу (2.11) из уравнения $(2.8 \mathrm{~b})$ получим

$$
\int_{0}^{1}(0, \tau) \Psi_{\bar{r}_{0}}(\tau) d \tau=0 .
$$

Из формул (2.7), (2.9) и (2.11) следует, что

$$
\begin{gathered}
\Psi_{\bar{r}_{0}}(\tau)=\left(\begin{array}{c}
\bar{r}_{0,3} \\
\tau^{2} \bar{r}_{0,1}-\tau \bar{r}_{0,2}+\bar{r}_{0,4}
\end{array}\right) \frac{1}{\left(\bar{r}_{0,3}^{2}+\left(\tau^{2} \bar{r}_{0,1}-\tau \bar{r}_{0,2}+\bar{r}_{0,4}\right)^{2}\right)^{1 / 2}}, \\
\left\|\bar{r}_{0}\right\|=1 .
\end{gathered}
$$

Поскольку знаменатель в правой части формулы (2.14) неотрицателен, то из уравнения (2.12) следует, что

$$
\bar{r}_{0,3}=0 \text {. }
$$


С учетом этого выпишем уравнения (2.12), (2.13):

$$
\begin{aligned}
& \int_{0}^{1} \operatorname{sign}\left(\tau^{2} \bar{r}_{0,1}-\tau \bar{r}_{0,2}+\bar{r}_{0,4}\right) d \tau=0 . \\
& \int_{0}^{1} \tau \operatorname{sign}\left(\tau^{2} \bar{r}_{0,1}-\tau \bar{r}_{0,2}+\bar{r}_{0,4}\right) d \tau=0 .
\end{aligned}
$$

Из уравнения (2.8а) получим

$$
-2 x_{0,1} \lim _{\varepsilon, \mu \rightarrow 0} \frac{\varepsilon^{6}}{T_{\varepsilon, \mu}^{3}}=\int_{0}^{1}\left(0, \tau^{2}\right) \Psi_{\bar{r}_{0}}(\tau) d \tau=\int_{0}^{1} \tau^{2} \operatorname{sign}\left(\tau^{2} \bar{r}_{0,1}-\tau \bar{r}_{0,2}+\bar{r}_{0,4}\right) d \tau .
$$

Утверждение 1. Система уравнений (2.15)-(2.19) имеет единственное решение.

Д о к а з а т е ль с т в о. Заметим, что все компоненты вектора $\bar{r}_{0}$ не могут быть нулевыми, иначе нарушится условие нормировки (2.15). В силу уравнения (2.17) многочлен $\tau^{2} \bar{r}_{0,1}-\tau \bar{r}_{0,2}+\bar{r}_{0,4}$ необходимо обращается в нуль в одной или двух точках из интервала $(0,1)$. Предположим сначала, что только один корень этого многочлена $\tau_{1}$ принадлежит интервалу $(0,1)$, тогда из $(2.17)$ вытекает, что подынтегральная функция меняет знак в середине отрезка интегрирования, т. е. $\tau_{1}=1 / 2$, но это противоречит уравнению (2.18). Значит, подынтегральная функция в (2.17) меняет знак в двух точках $\tau_{1}, \tau_{2} \in(0,1)$ и, как следствие,

$$
\bar{r}_{0,1} \neq 0 \text {. }
$$

Тогда уравнения (2.17), (2.18) преобразуются к виду

$$
\int_{0}^{\tau_{1}} d \tau-\int_{\tau_{1}}^{\tau_{2}} d \tau+\int_{\tau_{2}}^{1} d \tau=0, \quad \int_{0}^{\tau_{1}} \tau d \tau-\int_{\tau_{1}}^{\tau_{2}} \tau d \tau+\int_{\tau_{2}}^{1} \tau d \tau=0
$$

Эта система уравнений имеет единственное решение

$$
\tau_{1}=1 / 4, \quad \tau_{2}=3 / 4 .
$$

Тогда для

$$
a=\bar{r}_{0,2} / \bar{r}_{0,1}, \quad b=\bar{r}_{0,4} / \bar{r}_{0,1}
$$

получим

$$
a=1, \quad b=3 / 16 .
$$

Отметим, что $\bar{r}_{0,2} \neq 0$ и $\bar{r}_{0,4} \neq 0$. Далее, интеграл в (2.19) имеет вид

$$
\int_{0}^{1}\left(0, \tau^{2}\right) \Psi_{\bar{r}_{0}}(\tau) d \tau=\operatorname{sign}\left(\bar{r}_{0,1}\right) \int_{0}^{1} \tau^{2} \operatorname{sign}((\tau-1 / 4)(\tau-3 / 4)) d \tau=\operatorname{sign}\left(\bar{r}_{0,1}\right) / 16 \neq 0,
$$

следовательно,

$$
\lim _{\varepsilon, \mu \rightarrow 0} \frac{T_{\varepsilon, \mu}}{\varepsilon^{2}}=T_{0}>0 .
$$

Тогда из $(2.19),(2.24),(2.25)$ вытекает соотношение $-32 x_{0,1}=T_{0}^{3} \operatorname{sign}\left(\bar{r}_{0,1}\right)$. Отсюда и из $(2.15)$, (2.23) однозначно определяются $\bar{r}_{0,1}$ и $T_{0}$.

Утверждение 1 влечет справедливость следующего утверждения. 
Утверждение 2. Вектор $\bar{r}_{0}$ является единственной предельной точкой при $\varepsilon, \mu \rightarrow 0$ множества векторов $\bar{r}_{\varepsilon, \mu}$ с условием (2.9), и, тем самым, существует $\lim _{\varepsilon, \mu \rightarrow 0} \bar{r}_{\varepsilon, \mu}=\bar{r}_{0}$.

Для дальнейших рассуждений удобно ввести новую нормировку вектора $\bar{r}_{\varepsilon, \mu}$ при всех достаточно малых $\varepsilon$ и $\mu$ :

$$
\left|\bar{r}_{\varepsilon, \mu, 1}\right|=1, \quad\left|\bar{r}_{0,1}\right|=1 .
$$

При этом в силу (2.20) утверждения 1,2 останутся справедливыми.

Тогда при малых $\varepsilon, \mu$ выполняется $\bar{r}_{\varepsilon, \mu, 1} \cdot \bar{r}_{0,1}>0$ и, следовательно,

$$
\{-1,1\} \ni l:=\bar{r}_{\varepsilon, \mu, 1} \equiv \bar{r}_{0,1}=-\operatorname{sign}\left(x_{0,1}\right), \quad T_{0}=\sqrt[3]{32\left|x_{0,1}\right|} .
$$

Без ограничения общности далее будем полагать, что $l=1$.

\section{3. Алгоритм построения асимптотики решения}

Введем новые неизвестные малые величины $\theta_{\varepsilon, \mu}$ и $\rho_{\varepsilon, \mu}$ по формулам

$$
\vartheta_{\varepsilon, \mu}=\varepsilon\left(\vartheta_{0}+\theta_{\varepsilon, \mu}\right), \quad \bar{r}_{\varepsilon, \mu}=\bar{r}_{0}+\rho_{\varepsilon, \mu},
$$

где $\vartheta_{0}=T_{0}^{-1}$. Из (2.27) получаем

$$
\rho_{\varepsilon, \mu, 1}=0 .
$$

Задача, таким образом, сводится к нахождению асимптотики скалярной величины $\theta_{\varepsilon, \mu}$ и остальных компонент вектора $\rho_{\varepsilon, \mu}$. Обозначим

$$
\omega_{\varepsilon, \mu}=\left(\theta_{\varepsilon, \mu}, \rho_{\varepsilon, \mu}^{*}\right)^{*} .
$$

Принимая во внимание соотношения (2.7), (2.11), (2.22), (2.23), (2.26), (2.27), (3.1), запишем в новых обозначениях формулу для функции $\Psi_{\bar{r}_{\varepsilon, \mu}}(\tau)=\Psi_{\omega_{\varepsilon, \mu}}(\tau)$ :

$$
\begin{aligned}
& \Psi_{\omega_{\varepsilon, \mu}}(\tau)=\left(\begin{array}{c}
-2 \varepsilon\left(\vartheta_{0}+\theta_{\varepsilon, \mu}\right) \tau+\rho_{\varepsilon, \mu, 3} \\
\tau^{2}-\tau\left(1+\rho_{\varepsilon, \mu, 2}\right)+b+\rho_{\varepsilon, \mu, 4}
\end{array}\right) \\
& \times\left(\left(-2 \varepsilon\left(\vartheta_{0}+\theta_{\varepsilon, \mu}\right) \tau+\rho_{\varepsilon, \mu, 3}\right)^{2}+\left(\tau^{2}-\tau\left(1+\rho_{\varepsilon, \mu, 2}\right)+b+\rho_{\varepsilon, \mu, 4}\right)^{2}\right)^{-1 / 2}
\end{aligned}
$$

и систему (2.8) в матричной форме

$$
g\left(\varepsilon, \mu, \theta_{\varepsilon, \mu}\right)=\int_{0}^{1} G\left(\tau, \varepsilon, \theta_{\varepsilon, \mu}\right) \Psi_{\omega_{\varepsilon, \mu}}(\tau) d \tau=: \mathcal{G}\left(\varepsilon, \omega_{\varepsilon, \mu}\right) .
$$

Здесь

$$
g\left(\varepsilon, \mu, \theta_{\varepsilon, \mu}\right)=\left(\begin{array}{c}
-2\left(\vartheta_{0}+\theta_{\varepsilon, \mu}\right)^{3} x_{0,1} \\
\left(\vartheta_{0}+\theta_{\varepsilon, \mu}\right)^{2} \varepsilon^{2} \mu \xi \\
\varepsilon\left(\vartheta_{0}+\theta_{\varepsilon, \mu}\right)\left(\mu \xi-y_{0,1}\right) \\
-\varepsilon\left(\vartheta_{0}+\theta_{\varepsilon, \mu}\right) y_{0,2}
\end{array}\right), \quad G\left(\tau, \varepsilon, \theta_{\varepsilon, \mu}\right)=\left(\begin{array}{cc}
-2 \varepsilon\left(\vartheta_{0}+\theta_{\varepsilon, \mu}\right) \tau & \tau^{2} \\
0 & \tau \\
1 & 0 \\
0 & 1
\end{array}\right)
$$

Отметим, что $\mathcal{G}(\varepsilon, \omega)$ - правая часть уравнения (3.4) - в силу леммы 4 из [9] есть непрерывная по $\omega$ функция. Будем далее искать решение системы (3.4) среди векторов $\omega_{\varepsilon, \mu}$, для которых выполняется оценка

$$
\left\|\omega_{\varepsilon, \mu}\right\|=O^{*}(\varepsilon), \quad \varepsilon \rightarrow 0, \text { равномерно по } \mu \rightarrow 0 .
$$


Запись $\varphi(\varepsilon, \mu)=O^{*}\left(\varepsilon^{\gamma}\right), \varepsilon, \mu \rightarrow 0$ означает, что $\forall \sigma<\gamma \quad \varphi(\varepsilon, \mu)=o\left(\varepsilon^{\sigma}\right)$ [12]. В частности, $\varepsilon \ln ^{m}(\varepsilon), m>0$, есть $O^{*}(\varepsilon)$ при $\varepsilon, \mu \rightarrow 0$, но не $O(\varepsilon)$. Отметим, что при $\gamma>0$ выполняется $O^{*}\left(\varepsilon^{1+\gamma}\right)=O^{*}(\varepsilon) O\left(\varepsilon^{\gamma}\right)$.

Найдем асимптотическое разложение функции $\mathcal{G}(\varepsilon, \omega)$ при $(\varepsilon, \omega) \rightarrow 0$.

Разобьем отрезок интегрирования на две части, содержащие по одному корню (2.21) многочлена $\tau^{2}-\tau+3 / 16$ в знаменателе $\Psi_{\omega_{\varepsilon, \mu}}(\tau)(3.3)$, и представим интеграл в правой части системы (3.4) в виде

$$
\int_{0}^{1} G\left(\tau, \varepsilon, \theta_{\varepsilon, \mu}\right) \Psi_{\omega_{\varepsilon, \mu}}(\tau) d \tau=\int_{0}^{1 / 2}+\int_{1 / 2}^{1}=: J_{1}\left(\varepsilon, \omega_{\varepsilon, \mu}\right)+J_{2}\left(\varepsilon, \omega_{\varepsilon, \mu}\right) .
$$

Далее, для нахождения асимптотики интегралов $J_{1}$ и $J_{2}$ применим метод вспомогательного параметра, описанный в $[12 ; 13]$. Так, $J_{1}$ разбивается на сумму интегралов следующим образом:

$$
J_{1}\left(\varepsilon, \omega_{\varepsilon, \mu}\right)=\int_{0}^{1 / 4-\nu}+\int_{1 / 4-\nu}^{1 / 4+\nu}+\int_{1 / 4+\nu}^{1 / 2}=: J_{1,1}\left(\varepsilon, \omega_{\varepsilon, \mu}, \nu\right)+J_{1,2}\left(\varepsilon, \omega_{\varepsilon, \mu}, \nu\right)+J_{1,3}\left(\varepsilon, \omega_{\varepsilon, \mu}, \nu\right),
$$

где $\nu$ - малый вспомогательный параметр. Пусть

$$
\nu=\varepsilon^{p}, \quad p \in(0,1) .
$$

Асимптотики интегралов $J_{1,1}$ и $J_{1,3}$ получаются путем разложения подынтегральных функций в ряды с членами одного порядка малости по $\varepsilon$ и координатам вектора $\omega_{\varepsilon, \mu}$ с аналитическими по $\tau$ векторными коэффициентами. Тем самым для любого $N \geqslant 1$ имеем

$$
J_{1,1}\left(\varepsilon, \omega_{\varepsilon, \mu}, \nu\right)=\left[\int_{0}^{1 / 4-\nu} G_{0}(\tau) d \tau\right]_{0, \nu}\left(\begin{array}{l}
0 \\
1
\end{array}\right)+\sum_{k=1}^{N} \mathcal{F}_{k}\left(\varepsilon, \omega_{\varepsilon, \mu}\right)+\stackrel{1}{\mathfrak{F}}\left(\varepsilon, \omega_{\varepsilon, \mu}, \nu\right)+O^{*}\left(\varepsilon^{N+1}\right),
$$

где матрица $G_{0}(\tau)$ - слагаемое нулевого порядка малости по $\varepsilon$ и $\theta_{\varepsilon, \mu}$ в разложении $G\left(\tau, \varepsilon, \theta_{\varepsilon, \mu}\right)=$ $G_{0}(\tau)+\varepsilon G_{1}(\tau)+\varepsilon \theta_{\varepsilon, \mu} G_{2}(\tau)$, индексами $0, \nu$ отмечены слагаемые 0-го порядка малости по $\nu$, при этом

$$
\left[\int_{0}^{1 / 4-\nu} G_{0}(\tau) d \tau\right]_{0, \nu}=\int_{0}^{1 / 4} G_{0}(\tau) d \tau
$$

Кроме этого, в формуле $(3.8)$ и далее через $\stackrel{i}{\mathcal{F}}_{k}\left(\varepsilon, \omega_{\varepsilon, \mu}\right)(i=1, \ldots)$ обозначены векторы с компонентами - однородными многочленами степени $k$ относительно $\varepsilon$ и координат вектора $\omega_{\varepsilon, \mu}$ с известными коэффициентами, в частности

$$
\stackrel{1}{\mathcal{F}}_{1}\left(\varepsilon, \omega_{\varepsilon, \mu}\right)=\left(0,0,-2 \varepsilon \vartheta_{0} c_{1}+c_{0} \rho_{\varepsilon, \mu, 3}, 0\right)^{*}, \quad c_{0}=-2 \ln 2-2 \ln 3, \quad c_{1}=\frac{1}{2} \ln 2-\frac{3}{2} \ln 3,
$$

а через $\underset{\mathfrak{F}}{\mathfrak{F}}\left(\varepsilon, \omega_{\varepsilon, \mu}\right)$ обозначена сумма конечного числа слагаемых вида

$$
\varphi(\varepsilon) \psi\left(\omega_{\varepsilon, \mu}\right) \nu^{c} \ln ^{d} \nu, \quad d \in \mathbb{N} \cup\{0\}, \quad c^{2}+d^{2} \neq 0, \quad \varphi(\varepsilon)=O^{*}\left(\varepsilon^{\gamma_{1}}\right), \quad \psi\left(\omega_{\varepsilon, \mu}\right)=O^{*}\left(\left\|\omega_{\varepsilon, \mu}\right\|^{\gamma_{2}}\right)
$$

для некоторых $\gamma_{1}, \gamma_{2}$, что в силу предположения (3.5) дает $\psi\left(\omega_{\varepsilon, \mu}\right)=O^{*}\left(\varepsilon^{\gamma_{2}}\right)$. Здесь и далее символы $O^{*}, O, o$ будут использоваться для асимптотических оценок как векторных, так и скалярных величин (запись $\varphi=O^{*}\left(\varepsilon^{\sigma}\right)$ означает $\|\varphi\|=O^{*}\left(\varepsilon^{\sigma}\right)$ ). 
В силу леммы $\left[12\right.$, п. 2] при получении асимптотики интеграла $J_{1}\left(\varepsilon, \omega_{\varepsilon, \mu}\right)$ методом вспомогательного параметра слагаемые $\stackrel{j}{\mathfrak{F}}\left(\varepsilon, \omega_{\varepsilon, \mu}, \nu\right)$ можно не учитывать (в сумме (3.7) все слагаемые такого вида взаимно уничтожаются).

Аналогичным образом получаем для любого $N \geqslant 1$

$$
J_{1,3}\left(\varepsilon, \omega_{\varepsilon, \mu}, \nu\right)=-\int_{1 / 4}^{1 / 2} G_{0}(\tau) d \tau\left(\begin{array}{c}
0 \\
1
\end{array}\right)+\sum_{k=1}^{N} \stackrel{2}{\mathcal{F}}_{k}\left(\varepsilon, \omega_{\varepsilon, \mu}\right)+\stackrel{2}{\mathfrak{F}}\left(\varepsilon, \omega_{\varepsilon, \mu}, \nu\right)+O^{*}\left(\varepsilon^{N+1}\right) .
$$

В интеграле $J_{1,2}$ сделаем замену переменной интегрирования по формуле $\eta=\tau-1 / 4$ :

$$
\int_{1 / 4-\nu}^{1 / 4+\nu} G\left(\tau, \varepsilon, \theta_{\varepsilon, \mu}\right) \Psi_{\omega_{\varepsilon, \mu}}(\tau) d \tau=\int_{-\nu}^{\nu} \bar{G}\left(\eta, \varepsilon, \theta_{\varepsilon, \mu}\right) \bar{\Psi}_{\omega_{\varepsilon, \mu}}(\eta) d \eta
$$

При следующем дополнительном предположении о решении системы (3.2), (3.4)

$$
0 \neq-\varepsilon \vartheta_{0}+2 \rho_{\varepsilon, \mu, 3}=O^{*}(\varepsilon), \quad \forall \gamma>0 \quad \varepsilon^{1+\gamma}=o\left(-\varepsilon \vartheta_{0}+2 \rho_{\varepsilon, \mu, 3}\right), \quad \varepsilon, \mu \rightarrow 0,
$$

новая подынтегральная функция раскладывается в ряд с членами одного порядка малости по $\eta, \varepsilon$ и координатам вектора $\omega_{\varepsilon, \mu}$.

Далее, аналогично предшествующей работе авторов можно получить представление интеграла $J_{1,2}$ для любого $N \geqslant 1$ :

$$
J_{1,2}\left(\varepsilon, \omega_{\varepsilon, \mu}, \nu\right)=\sum_{k=1}^{N} \stackrel{3}{\mathcal{F}}_{k}\left(\varepsilon, \omega_{\varepsilon, \mu}\right)+\ln \left|b_{\varepsilon, \mu}-a_{\varepsilon, \mu}^{2}\right| \sum_{k=1}^{N} \stackrel{4}{\mathcal{F}}_{k}\left(\varepsilon, \omega_{\varepsilon, \mu}\right)+\stackrel{3}{\mathfrak{F}}\left(\varepsilon, \omega_{\varepsilon, \mu}, \nu\right)+O^{*}\left(\varepsilon^{N+1}\right),
$$

где, в частности,

$$
\begin{gathered}
\mathcal{F}_{1}\left(\varepsilon, \omega_{\varepsilon, \mu}\right)=-\widetilde{\rho}_{\varepsilon, \mu, 1}\left(\frac{1}{4}, 1,0,4\right)^{*}, \quad \mathcal{F}_{1}\left(\varepsilon, \omega_{\varepsilon, \mu}\right)=\left(0,0, \varepsilon \vartheta_{0}-2 \rho_{\varepsilon, \mu, 3}, 0\right)^{*}, \\
\widetilde{\rho}_{\varepsilon, \mu, 1}:=\rho_{\varepsilon, \mu, 2} / 4-\rho_{\varepsilon, \mu, 4}, \\
a_{\varepsilon, \mu}=2 \widetilde{\rho}_{\varepsilon, \mu, 1}, \quad b_{\varepsilon, \mu}=4 \widetilde{\rho}_{\varepsilon, \mu, 1}^{2}+\left(-\varepsilon \vartheta_{0}+2 \rho_{\varepsilon, \mu, 3}\right)^{2}, \quad b_{\varepsilon, \mu}-a_{\varepsilon, \mu}^{2}=\left(\varepsilon \vartheta_{0}-2 \rho_{\varepsilon, \mu, 3}\right)^{2} .
\end{gathered}
$$

Асимптотика интеграла $J_{2}\left(\varepsilon, \omega_{\varepsilon, \mu}\right)(3.6)$ строится аналогично $J_{1}\left(\varepsilon, \omega_{\varepsilon, \mu}\right)$. Выпишем здесь соотношение для интеграла $J_{2,2}\left(\varepsilon, \omega_{\varepsilon, \mu}, \nu\right)$, где $\nu$, вообще говоря, уже другой малый вспомогательный параметр. При дополнительных условиях

$$
0 \neq-3 \varepsilon \vartheta_{0}+2 \rho_{\varepsilon, \mu, 3}=O^{*}(\varepsilon), \quad \forall \gamma>0 \quad \varepsilon^{1+\gamma}=o\left(-3 \varepsilon \vartheta_{0}+2 \rho_{\varepsilon, \mu, 3}\right), \quad \varepsilon, \mu \rightarrow 0,
$$

для любого $N \geqslant 1$ справедливо

$$
J_{2,2}\left(\varepsilon, \omega_{\varepsilon, \mu}, \nu\right)=\sum_{k=1}^{N} \stackrel{5}{\mathcal{F}}_{k}\left(\varepsilon, \omega_{\varepsilon, \mu}\right)+\ln \left|\bar{b}_{\varepsilon, \mu}-\bar{a}_{\varepsilon, \mu}^{2}\right| \sum_{k=1}^{N} \stackrel{6}{\mathcal{F}}_{k}\left(\varepsilon, \omega_{\varepsilon, \mu}\right)+\stackrel{4}{\mathfrak{F}}\left(\varepsilon, \omega_{\varepsilon, \mu}, \nu\right)+O^{*}\left(\varepsilon^{N+1}\right),
$$

где, в частности,

$$
\stackrel{5}{\mathcal{F}}_{1}\left(\varepsilon, \omega_{\varepsilon, \mu}\right)=-\widetilde{\rho}_{\varepsilon, \mu, 2}\left(\frac{9}{4}, 3,0,4\right)^{*}, \quad \stackrel{6}{\mathcal{F}}_{1}\left(\varepsilon, \omega_{\varepsilon, \mu}\right)=\left(0,0,3 \varepsilon \vartheta_{0}-2 \rho_{\varepsilon, \mu, 3}, 0\right)^{*},
$$

$\widetilde{\rho}_{\varepsilon, \mu, 2}:=(3 / 4) \rho_{\varepsilon, \mu, 2}-\rho_{\varepsilon, \mu, 4}, \bar{a}_{\varepsilon, \mu}=-2 \widetilde{\rho}_{\varepsilon, \mu, 2}, \bar{b}_{\varepsilon, \mu}=4 \widetilde{\rho}_{\varepsilon, \mu, 2}^{2}+\left(3 \varepsilon \vartheta_{0}-2 \rho_{\varepsilon, \mu, 3}\right)^{2}$.

Подставляя в (3.6) выражение для $J_{1}(\varepsilon, \mu, \nu)$, собранное из формул (3.8), (3.9), (3.11), и соотношение для $J_{2}(\varepsilon, \mu, \nu)$, выписанное аналогичным образом, и отбрасывая слагаемые, содержащие вспомогательный параметр, приходим к справедливости следующего утверждения. 
Утверждение 3. Если $\omega_{\varepsilon, \mu}$ удовлетворлет условиям (3.5), (3.10), (3.12), то

$$
\begin{aligned}
\int_{0}^{1} G(\tau, \varepsilon & \left., \theta_{\varepsilon, \mu}\right) \Psi_{\omega_{\varepsilon, \mu}}(\tau) d \tau \stackrel{a s}{=} \int_{0}^{1} \operatorname{sign}\left(\tau^{2}-\tau+b\right) G_{0}(\tau) d \tau\left(\begin{array}{c}
0 \\
1
\end{array}\right)+\sum_{k=1}^{\infty}{ }_{\mathcal{F}}^{7}\left(\varepsilon, \omega_{\varepsilon, \mu}\right) \\
& +\ln \left|b_{\varepsilon, \mu}-a_{\varepsilon, \mu}^{2}\right| \sum_{k=1}^{\infty} \stackrel{4}{\mathcal{F}}_{k}\left(\varepsilon, \omega_{\varepsilon, \mu}\right)+\ln \left|\bar{b}_{\varepsilon, \mu}-\bar{a}_{\varepsilon, \mu}^{2}\right| \sum_{k=1}^{\infty} \stackrel{6}{\mathcal{F}}_{k}\left(\varepsilon, \omega_{\varepsilon, \mu}\right),
\end{aligned}
$$

где, в частности,

$$
\begin{gathered}
\mathcal{\mathcal { F }}_{1}\left(\varepsilon, \omega_{\varepsilon, \mu}\right)=\left(-\frac{1}{4} \widetilde{\rho}_{\varepsilon, \mu, 1}-\frac{9}{4} \widetilde{\rho}_{\varepsilon, \mu, 2},-\widetilde{\rho}_{\varepsilon, \mu, 1}-3 \widetilde{\rho}_{\varepsilon, \mu, 2},-2 \varepsilon \vartheta_{0} d_{1}+d_{0} \rho_{\varepsilon, \mu, 3},-4 \widetilde{\rho}_{\varepsilon, \mu, 1}-4 \widetilde{\rho}_{\varepsilon, \mu, 2}\right)^{*}, \\
d_{0}=-4(\ln 2+\ln 3), \quad d_{1}=\ln 2-2 \ln 3 .
\end{gathered}
$$

Это означает, что для любого $N \geqslant 1$ справедливо равенство

$$
\begin{gathered}
\int_{0}^{1} G\left(\tau, \varepsilon, \theta_{\varepsilon, \mu}\right) \Psi_{\omega_{\varepsilon, \mu}}(\tau) d \tau=\int_{0}^{1} \operatorname{sign}\left(\tau^{2}-\tau+b\right) G_{0}(\tau) d \tau\left(\begin{array}{c}
0 \\
1
\end{array}\right)+\sum_{k=1}^{N} \stackrel{7}{\mathcal{F}}_{k}\left(\varepsilon, \omega_{\varepsilon, \mu}\right) \\
+\ln \left|b_{\varepsilon, \mu}-a_{\varepsilon, \mu}^{2}\right| \sum_{k=1}^{N} \frac{4}{\mathcal{F}_{k}}\left(\varepsilon, \omega_{\varepsilon, \mu}\right)+\ln \left|\bar{b}_{\varepsilon, \mu}-\bar{a}_{\varepsilon, \mu}^{2}\right| \sum_{k=1}^{N}{ }_{\mathcal{F}_{k}}^{6}\left(\varepsilon, \omega_{\varepsilon, \mu}\right)+O^{*}\left(\varepsilon^{N+1}\right), \quad \varepsilon, \mu \rightarrow 0 .
\end{gathered}
$$

Подставив в правую часть (3.4) разложение интеграла (3.13), с учетом соотношений (2.15)-(2.19) для уже найденных $\vartheta_{0}=T_{0}^{-1}, \bar{r}_{0}$ выпишем систему для неизвестных малых компонент вектора $\omega_{\varepsilon, \mu}$ :

$$
g_{1}\left(\varepsilon, \mu, \theta_{\varepsilon, \mu}\right)=\sum_{k=1}^{\infty} \stackrel{7}{\mathcal{F}}_{k}\left(\varepsilon, \omega_{\varepsilon, \mu}\right)+\ln \left|b_{\varepsilon, \mu}-a_{\varepsilon, \mu}^{2}\right| \sum_{k=1}^{\infty} \stackrel{4}{\mathcal{F}}_{k}\left(\varepsilon, \omega_{\varepsilon, \mu}\right)+\ln \left|\bar{b}_{\varepsilon, \mu}-\bar{a}_{\varepsilon, \mu}^{2}\right| \sum_{k=1}^{\infty}{ }_{\mathcal{F}}^{6}{ }_{k}\left(\varepsilon, \omega_{\varepsilon, \mu}\right),
$$

где $g_{1}\left(\varepsilon, \mu, \theta_{\varepsilon, \mu}\right)=g\left(\varepsilon, \mu, \theta_{\varepsilon, \mu}\right)-\left(-2 \vartheta_{0}^{3} x_{0,1}, 0,0,0\right)^{*}$.

\section{4. Получение первых асимптотических приближений решения}

Оставляя в (3.14) слагаемые первого порядка малости по $\varepsilon$ и $\mu$, получим систему первого приближения относительно неизвестных малых величин $\rho_{1, k}, k=\overline{2,4}$ и $\theta_{1}$ (здесь и далее первый индекс 1 означает первое приближение).

$$
\begin{gathered}
6 \theta_{1} \vartheta_{0}^{2} x_{0,1}-\frac{7}{4} \rho_{1,2}+\frac{5}{2} \rho_{1,4}=0, \\
-\frac{5}{2} \rho_{1,2}+4 \rho_{1,4}=0, \\
d_{0} \rho_{1,3}+2 \alpha\left(\varepsilon \vartheta_{0}-2 \rho_{1,3}\right)+2 \beta\left(3 \varepsilon \vartheta_{0}-2 \rho_{1,3}\right)=\varepsilon \vartheta_{0}\left(-y_{0,1}+2 d_{1}\right), \\
-4 \rho_{1,2}+8 \rho_{1,4}=-\varepsilon \vartheta_{0} y_{0,2}, \\
\alpha=\ln \left|\varepsilon \vartheta_{0}-2 \rho_{1,3}\right|, \quad \beta=\ln \left|3 \varepsilon \vartheta_{0}-2 \rho_{1,3}\right| .
\end{gathered}
$$

Отметим, что малый параметр $\mu$ не входит в уравнения системы (4.1), следовательно, компоненты первого приближения решения будут функциями только от $\varepsilon$. Из уравнений (4.1a), (4.1b), (4.1d) найдем

$$
\rho_{1,2}=-\varepsilon \vartheta_{0} y_{0,2}, \quad \rho_{1,4}=-\varepsilon \frac{5}{8} \vartheta_{0} y_{0,2}, \quad \theta_{1}=\varepsilon \frac{3 y_{0,2}}{96 \vartheta_{0} x_{0,1}} .
$$

Заметим, что для этих величин справедлива оценка $O(\varepsilon), \varepsilon, \mu \rightarrow 0$. 
Утверждение 4. При всех достаточно малых $\varepsilon>0$ уравнение (4.1c) имеет единственное решение $\rho_{1,3}=\rho_{1,3}(\varepsilon)$, удовлетворяющее неравенствам

$$
\frac{3}{4} \vartheta_{0} \varepsilon<\rho_{1,3}<\frac{5}{4} \vartheta_{0} \varepsilon
$$

Д о к а з а т е л ь с т в о. Представим $\rho_{1,3}$ в виде

$$
\rho_{1,3}:=\frac{1}{2} \varepsilon \vartheta_{0} \bar{\rho}
$$

и выпишем уравнение (4.1c) относительно новой неизвестной величины $\bar{\rho}$ :

$$
0=y_{0,1}-2 d_{1}+\frac{d_{0}}{2} \bar{\rho}+4(2-\bar{\rho}) \ln \left(\varepsilon \vartheta_{0}\right)+2(1-\bar{\rho}) \ln |1-\bar{\rho}|+2(3-\bar{\rho}) \ln |3-\bar{\rho}|=: f(\bar{\rho}, \varepsilon) .
$$

Функция $f(\bar{\rho}, \varepsilon)$ обладает следующими свойствами:

1. $\forall \varepsilon>0$ функция $f(\bar{\rho}, \varepsilon)$ непрерывна по $\bar{\rho}$ на $(1,3)$.

2. $\exists \varepsilon_{1}>0 \forall \varepsilon \in\left(0, \varepsilon_{1}\right]$ выполняется $f(3 / 2, \varepsilon)<0$, поскольку

$$
f(3 / 2, \varepsilon)=y_{0,1}-2 d_{1}+\frac{3 d_{0}}{4}+\ln 2+3 \ln \frac{3}{2}+2 \ln \left(\varepsilon \vartheta_{0}\right) \longrightarrow-\infty, \quad \varepsilon \rightarrow+0 .
$$

3. $\exists \varepsilon_{2}>0 \forall \varepsilon \in\left(0, \varepsilon_{2}\right]$ выполняется $f(5 / 2, \varepsilon)>0$, поскольку

$$
f(5 / 2, \varepsilon)=y_{0,1}-2 d_{1}+\frac{5 d_{0}}{4}-3 \ln \frac{3}{2}-\ln 2-2 \ln \left(\varepsilon \vartheta_{0}\right) \longrightarrow+\infty, \quad \varepsilon \rightarrow+0 .
$$

Из свойств 1-3 следует, что $\forall \varepsilon, 0<\varepsilon<\min \left\{\varepsilon_{1}, \varepsilon_{2}\right\} \exists \bar{\rho}_{\varepsilon} \in(3 / 2,5 / 2)$, что $f\left(\bar{\rho}_{\varepsilon}, \varepsilon\right)=0$.

Далее,

4. $\forall \varepsilon>0$ функция $f(\bar{\rho}, \varepsilon)$ дифференцируема по $\bar{\rho}$ на $(1,3)$, значит, и на $[3 / 2,5 / 2]$, при этом

$$
\frac{\partial}{\partial \bar{\rho}} f(\bar{\rho}, \varepsilon)=\frac{d_{0}}{2}-4-2 \ln |1-\bar{\rho}|-2 \ln |3-\bar{\rho}|-4 \ln \left(\varepsilon \vartheta_{0}\right) \rightrightarrows+\infty, \quad \bar{\rho} \in[3 / 2,5 / 2], \quad \varepsilon \rightarrow+0 .
$$

Следовательно, при всех достаточно малых $\varepsilon$ функция $f(\bar{\rho}, \varepsilon)$ строго возрастает по $\bar{\rho}$ на $[3 / 2,5 / 2]$, значит, решение $\bar{\rho}_{\varepsilon} \in(3 / 2,5 / 2)$ единственное.

Подставив $\bar{\rho}_{\varepsilon}$ в формулу (4.3), получим единственное решение $\rho_{1,3}$ уравнения (4.1c), удовлетворяющее неравенствам (4.2).

Утверждение 5. Пусть выполнено условие

$$
2 d_{1}-d_{0}-y_{0,1} \neq 0
$$

Тогда при всех достаточно малых $\varepsilon>0$ решение $\rho_{1,3}$ уравнения (4.1c) имеет вид

$$
\rho_{1,3}=\vartheta_{0} \varepsilon+\varphi(\varepsilon), \quad \varphi(\varepsilon)=o(\varepsilon), \quad \varphi(\varepsilon) \neq O\left(\varepsilon^{1+\sigma}\right), \forall \sigma \in(0,1), \quad \varepsilon \rightarrow+0 .
$$

Д о к а з а т е л ь с т в о. Из доказательства утверждения 4 следует, что множество значений решения уравнения (4.4) $\{\bar{\rho}(\varepsilon)\}$ ограничено при всех достаточно малых $\varepsilon$, следовательно, имеет предельные точки при $\varepsilon \rightarrow 0$. Рассмотрим произвольную $\gamma$ из них. Опуская индекс $n$ у членов соответствующей последовательности $\varepsilon_{n}$, представим $\bar{\rho}(\varepsilon)$ в виде $\bar{\rho}(\varepsilon)=\gamma+\bar{\varphi}(\varepsilon)$, где $\gamma \in \mathbb{R}, \bar{\varphi}(\varepsilon)=o(1), \varepsilon \rightarrow 0$. Справедливо тождество

$$
\begin{aligned}
& 0 \underset{\varepsilon \in\left(0, \varepsilon_{0}\right]}{\equiv} y_{0,1}-2 d_{1}+\frac{d_{0}}{2}(\gamma+\bar{\varphi}(\varepsilon))+4(2-\gamma-\bar{\varphi}(\varepsilon)) \ln \left(\varepsilon \vartheta_{0}\right) \\
& +2(1-\gamma-\bar{\varphi}(\varepsilon)) \ln |1-\gamma-\bar{\varphi}(\varepsilon)|+2(3-\gamma-\bar{\varphi}(\varepsilon)) \ln |3-\gamma-\bar{\varphi}(\varepsilon)| .
\end{aligned}
$$

В силу утверждения 4 выполняется $\gamma+\bar{\varphi}(\varepsilon) \in(3 / 2,5 / 2), \varepsilon \in\left(0, \varepsilon_{0}\right]$. Следовательно, $\gamma \in[3 / 2,5 / 2]$. Поэтому 


$$
y_{0,1}-2 d_{1}+d_{0}(\gamma+\bar{\varphi}(\varepsilon)) / 2+2(1-\gamma-\bar{\varphi}(\varepsilon)) \ln |1-\gamma-\bar{\varphi}(\varepsilon)|+2(3-\gamma-\bar{\varphi}(\varepsilon)) \ln |3-\gamma-\bar{\varphi}(\varepsilon)|
$$

ограничено при всех $\varepsilon \in\left(0, \varepsilon_{0}\right]$. Но если $\gamma \neq 2$, то оставшееся слагаемое в правой части (4.6) $4(2-\gamma-\bar{\varphi}(\varepsilon)) \ln \left(\varepsilon \vartheta_{0}\right)$ неограничено при $\varepsilon \rightarrow+0$. Поэтому $\gamma=2-$ единственная предельная точка множества $\{\bar{\rho}(\varepsilon)\}$ при $\varepsilon \rightarrow 0$.

В этом случае

$$
\begin{aligned}
& 4 \bar{\varphi}(\varepsilon) \ln \varepsilon \underset{\varepsilon \in\left(0, \varepsilon_{0}\right]}{\equiv} y_{0,1}-2 d_{1}+d_{0}+\frac{d_{0}}{2} \bar{\varphi}(\varepsilon)-4 \bar{\varphi}(\varepsilon) \ln \vartheta_{0} \\
& -2(1+\bar{\varphi}(\varepsilon)) \ln |1+\bar{\varphi}(\varepsilon)|+2(1-\bar{\varphi}(\varepsilon)) \ln |1-\bar{\varphi}(\varepsilon)| .
\end{aligned}
$$

Выражение в правой части (4.7) при $\varepsilon \rightarrow+0$ стремится к $y_{0,1}-2 d_{1}+d_{0}$, тогда в силу $(4.5)$ и $4 \bar{\varphi}(\varepsilon) \ln \varepsilon \longrightarrow y_{0,1}-2 d_{1}+d_{0} \neq 0$, т. е. $\bar{\varphi}(\varepsilon) \sim\left(y_{0,1}-2 d_{1}+d_{0}\right) /(4 \ln \varepsilon)=o(1), \varepsilon \rightarrow+0$, но $\bar{\varphi}(\varepsilon) \neq O\left(\varepsilon^{\sigma}\right) \forall \sigma \in(0,1)$. Далее, из (4.3) получим $\rho_{1,3}=\vartheta_{0} \varepsilon+\varphi(\varepsilon), \varphi(\varepsilon):=\vartheta_{0} \varepsilon \bar{\varphi}(\varepsilon) / 2$, $\varphi(\varepsilon)=o(\varepsilon)$, но $\varphi(\varepsilon) \neq O\left(\varepsilon^{1+\sigma}\right) \forall \sigma \in(0,1)$.

Таким образом, доказано существование решения системы первого приближения и получена его оценка $O(\varepsilon), \varepsilon, \mu \rightarrow 0$. При этом компонента $\rho_{1,3}$ решения, определяющаяся из уравнения (4.1c), как и в [6], имеет сложную структуру.

Аналогично [6] можно показать, что $\rho_{1,3}$ не является рациональной функцией от $\varepsilon$ и $\ln \varepsilon$.

В силу утверждений 4, 5 условия (3.5), (3.10), (3.12) в первом приближении выполняются.

\section{5. Получение и обоснование асимптотики оптимального времени $T_{\varepsilon, \mu}$ и оптимального управления $u_{\varepsilon, \mu}(t)$}

Будем искать величины $\theta_{\varepsilon, \mu}$ и $\rho_{\varepsilon, \mu}(3.1),(3.2)$ в виде

$$
\begin{gathered}
\theta_{\varepsilon, \mu}=\theta_{1}(\varepsilon)+\sum_{k=2}^{\infty} \theta_{k}(\varepsilon, \mu), \quad \rho_{\varepsilon, \mu}=\rho_{1}(\varepsilon)+\sum_{k=2}^{\infty} \rho_{k}(\varepsilon, \mu), \\
\theta_{k}(\varepsilon, \mu),\left\|\rho_{k}(\varepsilon, \mu)\right\|=O^{*}(\varepsilon) O\left(\varepsilon^{k-1}+\mu^{k-1}\right), \quad k \geqslant 2, \quad \varepsilon, \mu \rightarrow 0 .
\end{gathered}
$$

Отметим, что

$$
O^{*}(\varepsilon) O\left(\varepsilon^{n}+\mu^{n}\right) \times O^{*}(\varepsilon) O\left(\varepsilon^{k}+\mu^{k}\right)=O^{*}(\varepsilon) O\left(\varepsilon^{n+k+1}+\mu^{n+k+1}\right) .
$$

Члены $\omega_{k}=\left(\theta_{k}, \rho_{k}^{*}\right)^{*}, k=2, \ldots$ асимптотического разложения $\omega_{\varepsilon, \mu}$, находятся последовательно из систем, полученных путем подстановки представлений (5.1) в (3.14), и посредством приравнивания слагаемых одного порядка малости по $\varepsilon$ и $\mu$. Вид оценок (5.2) продиктован тем, что малый параметр $\mu$ входит в систему (5.2) только в произведении с $\varepsilon$.

Пусть $\rho_{\varepsilon, \mu, 3}=\rho_{1,3}+\widehat{\rho}_{3}$, где $\rho_{1,3}-$ найденная компонента решения системы первого приближения, $\widehat{\rho}_{3}=o\left(\rho_{1,3}\right), \varepsilon, \mu \rightarrow 0$. Разложим $\ln \left|b_{\varepsilon, \mu}-a_{\varepsilon, \mu}^{2}\right|$ и $\ln \left|\bar{b}_{\varepsilon, \mu}-\bar{a}_{\varepsilon, \mu}^{2}\right|$ в ряд на первом приближении аналогично работе [6]. С учетом обозначений (4.1е) получим

$$
\begin{aligned}
& \ln \left|b_{\varepsilon, \mu}-a_{\varepsilon, \mu}^{2}\right|=\ln \left(\varepsilon \vartheta_{0}-2 \rho_{1,3}-2 \widehat{\rho}_{3}\right)^{2}=2 \alpha-\frac{4 \widehat{\rho}_{3}}{W_{1}(\varepsilon)}+\sum_{k=2}^{\infty} \stackrel{8}{\mathcal{F}_{k}}\left(\frac{\widehat{\rho}_{3}}{W_{1}(\varepsilon)}\right), \\
& \ln \left|\bar{b}_{\varepsilon, \mu}-\bar{a}_{\varepsilon, \mu}^{2}\right|=\ln \left(3 \varepsilon \vartheta_{0}-2 \rho_{1,3}-2 \widehat{\rho}_{3}\right)^{2}=2 \beta-\frac{4 \widehat{\rho}_{3}}{W_{2}(\varepsilon)}+\sum_{k=2}^{\infty} \stackrel{9}{\mathcal{F}}_{k}\left(\frac{\widehat{\rho}_{3}}{W_{2}(\varepsilon)}\right),
\end{aligned}
$$

где $W_{1}(\varepsilon):=\varepsilon \vartheta_{0}-2 \rho_{1,3}, W_{2}(\varepsilon):=3 \varepsilon \vartheta_{0}-2 \rho_{1,3}$.

Подставив эти ряды для логарифмов в (3.14) и разложив в ряды множители при них, для $\rho_{2, k}, k=\overline{2,4}$ и $\theta_{2}(5.1)$ получим однозначно разрешимую систему линейных уравнений 
с коэффициентами и правыми частями, явно зависящими от найденной из системы первого приближения компоненты $\rho_{1,3}$, не являющейся рациональной функцией от $\varepsilon$ и $\ln \varepsilon$, и от $\alpha, \beta$ (4.1е), в свою очередь зависящих от $\rho_{1,3}$ :

$$
\begin{aligned}
6 \theta_{2} \vartheta_{0}^{2} x_{0,1}-\frac{7}{4} \rho_{2,2}+\frac{5}{2} \rho_{2,4}=\stackrel{1}{\mathcal{P}}_{2}\left(\varepsilon, \omega_{1}\right)+\stackrel{1}{\mathcal{P}}_{2, \alpha}\left(\varepsilon, \omega_{1}\right) \alpha+\stackrel{1}{\mathcal{P}}_{2, \beta}\left(\varepsilon, \omega_{1}\right) \beta, \\
-\frac{5}{2} \rho_{2,2}+4 \rho_{2,4}=\stackrel{2}{\mathcal{P}}_{2}\left(\varepsilon, \omega_{1}\right)+\stackrel{2}{\mathcal{P}}_{2, \alpha}\left(\varepsilon, \omega_{1}\right) \alpha+\stackrel{2}{\mathcal{P}}_{2, \beta}\left(\varepsilon, \omega_{1}\right) \beta \\
\left(d_{0}-8-4 \alpha-4 \beta\right) \rho_{2,3}=\varepsilon \mu \vartheta_{0} \xi+\stackrel{3}{\mathcal{P}}_{2}\left(\varepsilon, \omega_{1}\right)+\stackrel{3}{\mathcal{P}}_{2, \alpha}\left(\varepsilon, \omega_{1}\right) \alpha+\stackrel{3}{\mathcal{P}}_{2, \beta}\left(\varepsilon, \omega_{1}\right) \beta, \\
-4 \rho_{2,2}+8 \rho_{2,4}=\stackrel{4}{\mathcal{P}_{2}}\left(\varepsilon, \omega_{1}\right)+\stackrel{4}{\mathcal{P}}_{2, \alpha}\left(\varepsilon, \omega_{1}\right) \alpha+\stackrel{4}{\mathcal{P}}_{2, \beta}\left(\varepsilon, \omega_{1}\right) \beta
\end{aligned}
$$

здесь $\stackrel{j}{\mathcal{P}}_{2}, \stackrel{j}{\mathcal{P}}_{2, \alpha}, \stackrel{j}{\mathcal{P}}_{2, \beta}, j=\overline{1,4}$ - известные однородные многочлены 2 -й степени по своим аргументам. Определитель $\operatorname{det} \mathcal{L}(\varepsilon)$ матрицы системы (5.4) отличен от нуля, следовательно, система однозначно разрешима. Обратная матрица $\mathcal{L}^{-1}(\varepsilon)$ ограничена равномерно по малым $\varepsilon$, тогда с учетом вида правых частей системы (5.4) получаем, что для ее решения справедлива оценка $\omega_{2}=\left(\theta_{2}, \rho_{2}^{*}\right)^{*}=O^{*}(\varepsilon) O(\varepsilon+\mu), \varepsilon, \mu \rightarrow 0$. При этом компоненты вектора $\omega_{2}$ не являются рациональными функциями от $\varepsilon$ и $\ln \varepsilon$, условия (3.5), (3.10), (3.12) для $\hat{\omega}_{2}=\omega_{1}+\omega_{2}$ выполняются. Отметим, что в подсистему для $\theta_{2}, \rho_{2,2}$ и $\rho_{2,4}$ не входит $\mu$, поэтому, в частности, $\theta_{2}$ зависит только от $\varepsilon$. Следующие члены асимптотики (5.1) находятся из соответствующих линейных систем с той же самой матрицей, что и у (5.4), а вид правых частей определяет справедливость оценок (5.2).

Пусть $\hat{\omega}_{N}=\sum_{k=1}^{N} \omega_{k}, \hat{\omega}=\omega_{\varepsilon, \mu}-\hat{\omega}_{N}$. Будем искать $\hat{\omega}$ в таком классе, что для $\omega_{\varepsilon, \mu}$ выполняются условия (3.5), (3.10), (3.12). В силу утверждения 3 с учетом определения $\omega_{i}, i=\overline{1, N}$ и соотношения (5.3) система (3.4) эквивалентна системе для $\hat{\omega}$ следующего вида

$$
\mathcal{L} \hat{\omega}=\mathcal{H}_{N+1}(\varepsilon, \mu, \hat{\omega}),
$$

где $\left\|\mathcal{H}_{N+1}(\varepsilon, \mu, \hat{\omega})\right\|=O^{*}(\varepsilon) O\left(\varepsilon^{N}+\mu^{N}\right)+O(\varepsilon\|\hat{\omega}\| \alpha)+O(\varepsilon\|\hat{\omega}\| \beta)+O\left(\|\hat{\omega}\|^{2} \alpha\right)+O\left(\|\hat{\omega}\|^{2} \beta\right)+$ $O\left(\frac{\varepsilon^{2}\|\hat{\omega}\|}{W_{1}(\varepsilon)}\right)+O\left(\frac{\varepsilon^{2}\|\hat{\omega}\|}{W_{2}(\varepsilon)}\right), \quad \varepsilon, \mu \rightarrow 0$. В силу ограниченнности $\mathcal{L}^{-1}(\varepsilon)$ равномерно по малым $\varepsilon$ и справедливости условий (3.5), (3.10) система (5.5) эквивалентна следующей системе:

$$
\hat{\omega}=F(\varepsilon, \mu, \hat{\omega}),
$$

где для любых $\varepsilon>0, \mu>0$ отображение $F(\varepsilon, \mu, \hat{\omega})$ непрерывно по $\hat{\omega}$ и при $\hat{\omega}=O^{*}(\varepsilon)$ выполняется $F(\varepsilon, \mu, \hat{\omega})=o(1)\|\hat{\omega}\|+O^{*}(\varepsilon) O\left(\varepsilon^{N}+\mu^{N}\right), \varepsilon, \mu \rightarrow 0$. Найдем компактное выпуклое множество, образ которого при отображении $F(\varepsilon, \mu, \hat{\omega})$ лежит в нем. Тогда по теореме Шаудера - Тихонова $[14$, с. 628$]$ у $F(\varepsilon, \mu, \hat{\omega})$ есть неподвижная точка в этом компакте. Рассмотрим $B\left[0, \varepsilon^{\gamma}\left(\varepsilon^{N}+\mu^{N}\right)\right]$ - шар в пространстве $\mathbb{R}^{5}$ с центром в нуле радиуса $\varepsilon^{\gamma}\left(\varepsilon^{N}+\mu^{N}\right), 0<\gamma<1$. Пусть $\|\hat{\omega}\| \leqslant \varepsilon^{\gamma}\left(\varepsilon^{N}+\mu^{N}\right)$. Тогда $\|F(\varepsilon, \mu, \hat{\omega})\| \leqslant\left(o(1)+O^{*}\left(\varepsilon^{1-\gamma}\right)\right) \varepsilon^{\gamma}\left(\varepsilon^{N}+\mu^{N}\right) \leqslant \varepsilon^{\gamma}\left(\varepsilon^{N}+\mu^{N}\right)$ для всех достаточно малых $\varepsilon$ и $\mu$. Поэтому уравнение (5.6) имеет решение $\hat{\omega}=O\left(\varepsilon^{\gamma}\left(\varepsilon^{N}+\mu^{N}\right)\right)$, $0<\gamma<1, \varepsilon, \mu \rightarrow 0$.

В силу единственности оптимального управления $u_{\varepsilon, \mu}(t)$ и времени быстродействия $T_{\varepsilon, \mu}$ асимптотические разложения для них получаются подстановкой найденных рядов для $\theta_{\varepsilon, \mu}$ и $\rho_{\varepsilon, \mu}$ с учетом замены (2.5) в формулы $(2.2),(2.11),(3.1)$. При этом непосредственными вычислениями получим, что $T_{1}, T_{2}$ и $r_{1}$ не зависят от $\mu$. Таким образом, справедлива следующая теорема.

Теорема. При выполнении условий (2.10), (3.10), (3.12) время быстродействия $T_{\varepsilon, \mu} u$ вектор начальных условий сопряженной системы $r_{\varepsilon, \mu}$ раскладываются в асимптотические ряды вида $T_{\varepsilon, \mu} \sim \varepsilon^{2}\left(T_{0}+T_{1}(\varepsilon)+T_{2}(\varepsilon)+\sum_{k=3}^{\infty} T_{k}(\varepsilon, \mu)\right), r_{\varepsilon, \mu} \sim r_{0}+r_{1}(\varepsilon)+\sum_{k=2}^{\infty} r_{k}(\varepsilon, \mu)$, где компоненты $T_{k}, r_{k}$ не являются рациональными функииями $\varepsilon u \ln \varepsilon$, при этом $T_{k}(\varepsilon, \mu)=O^{*}(\varepsilon) O\left(\varepsilon^{k-1}+\mu^{k-1}\right), r_{k}(\varepsilon, \mu)=O^{*}(\varepsilon) O\left(\varepsilon^{k-1}+\mu^{k-1}\right), \varepsilon, \mu \rightarrow 0$. 


\section{СПИСОК ЛИТЕРАТУРЫ}

1. Понтрягин Л.С., Болтянский В.Г., Гамкрелидзе Р.В., Мищенко Е.Ф. Математическая теория оптимальных процессов. М.: Физматгиз, 1961. 391 с.

2. Дмитриев М.Г., Курина Г.А. Сингулярные возмущения в задачах управления // Автоматика и телемеханика. 2006. №. 1. С. 3-51.

3. Kokotovic P.V., Haddad A.H. Controllability and time-optimal control of systems with slow and fast modes // IEEE Trans. Automat. Control. 1975. Vol. 20, no. 1. P. 111-113. doi: 10.1109/TAC.1975.1100852 .

4. Дончев А. Системы оптимального управления: Возмущения, приближения и анализ чувствительности. М.: Мир, 1987. 156 с.

5. Гичев Т.P., Дончев А.Л. Сходимость решения линейной сингулярно возмущенной задачи быстродействия // Прикл. математика и механика. 1979. Т. 43, № 3. С. 466-474.

6. Данилин А.P., Ильин А.М. О структуре решения одной возмущенной задачи быстродействия // Фундамент. и прикл. математика. 1998. Т. 4, № 3. С. 905-926.

7. Данилин А.Р., Парышева Ю.В. Асимптотика оптимального значения функционала качества в линейной задаче оптимального управления // Докл. АН. 2009. Т. 427, № 2. С. 151-154.

8. Шабуров А.А. Асимптотическое разложение решения сингулярно возмущенной задачи оптимального управления с интегральным выпуклым критерием качества и гладкими геометрическими ограничениями на управление // Изв. Ин-та математики и информатики Удмурт. гос. ун-та. 2017. Т. 50, № 2. С. 110-120.

9. Данилин А.P., Коврижных О.О. О зависимости задачи быстродействия для линейной системы от двух малых параметров // Вест. ЧелГУ. 2011. № 27. С. 46-60. (Математика, механика, информатика; вып. 14.)

10. Данилин А.P., Коврижных О.О. О задаче управления точкой малой массы в среде без сопротивления // Докл. АН. 2013. Т. 451, № 6. С. 612-614.

11. Ли Э.Б., Маркус Л. Основы теории оптимального управления. М.: Наука, 1972. 576 с.

12. Данилин А.P. Асимптотика оптимального значения функционала качества при быстростабилизирующемся непрямом управлении в сингулярном случае // Журн. вычисл. математики и мат. физики. 2006. Т. 46, № 12. С. 2166-2177.

13. Ильин А.М., Данилин А.Р. Асимптотические методы в анализе. М.: Физматлит, 2009.248 с. ISBN: 978-5-9221-1056-3 .

14. Канторович Л.В., Акилов Г.П. Функциональный анализ. М.: Наука. Гл. ред. физ.-мат. лит., 1984. 752 c.

Данилин Алексей Руфимович

Поступила 10.01.2019

После доработки 4.02.2019

Принята к публикации 11.02.2019

д-р физ.-мат. наук, профессор,

зав. отделом,

Институт математики и механики им. Н.Н. Красовского УрО РАН;

Уральский федеральный университет

г. Екатеринбург

e-mail: dar@imm.uran.ru

Коврижных Ольга Олеговна, канд. физ.-мат. наук, старший науч. сотрудник

Институт математики и механики им. Н.Н. Красовского УрО РАН,

Уральский федеральный университет

г. Екатеринбург

e-mail: koo@imm.uran.ru

\section{REFERENCES}

1. Pontryagin L.S., Boltyanskii V.G., Gamkrelidze R.V., Mishchenko E.F. The mathematical theory of optimal processes, ed. L.W. Neustadt, N Y; London, Interscience Publ. John Wiley \& Sons, Inc., 
1962, 360 p. ISBN: 0470693819 . Original Russian text published in Pontryagin L.S., Boltyanskii V.G., Gamkrelidze R.V., Mishchenko E.F. Matematicheskaya teoriya optimal'nykh protsessov, Moscow: Fizmatgiz Publ., 1961, 391 p.

2. Dmitriev M.G., Kurina G.A. Singular perturbations in control problems. Automation and Remote Control, 2006, vol. 67, no. 1, pp. 1-43. doi: 10.1134/S0005117906010012.

3. Kokotovic P.V., Haddad A.H. Controllability and time-optimal control of systems with slow and fast modes. IEEE Trans. Automat. Control., 1975, vol. 20, no. 1, pp. 111-113. doi: 10.1109/TAC.1975.1100852.

4. Dontchev A.L. Perturbations, approximations and sensitivity analisis of optimal control systems, Berlin; Heidelberg; N Y; Tokio: Springer-Verlag, 1983, 161 p. doi: 10.1007/BFb0043612. Translated to Russian under the title Sistemy optimal'nogo upravleniya: Vozmushcheniya, priblizheniya $i$ analiz chuvstvitel'nosti, Moscow: Mir Publ., 1987, 156 p.

5. Gichev T.R., Donchev A.L. Convergence of the solution of the linear singularly perturbed problem of time-optimal response. J. Appl. Math. Mech., 1979, vol. 43, no. 3, pp. 502-511. doi: 10.1016/0021-8928(79)90098-4.

6. Danilin A.R., Il'in A.M. On the structure of the solution of a perturbed optimal-time control problem. Fundament. Prikl. Matematika, 1998, vol. 4, no. 3, pp. 905-926 (in Russian).

7. Danilin A.R., Parysheva Y.V. Asymptotics of the optimal cost functional in a linear optimal control problem. Dokl. Math., 2009, vol. 80, no. 1, pp. 478-481. doi: 10.1134/S1064562409040073.

8. Shaburov A.A. Asymptotic expansion of a solution for the singularly perturbed optimal control problem with a convex integral quality index and smooth control constraints Proc. of the Institute of Math. and Inf. at Udmurt State University, 2017, vol. 50, no 2, pp. 110-120.

9. Danilin A.R., Kovrizhnykh O.O. On the dependence of the time-optimal control problem for a linear system of two small parameters. Vest. Chelyabinsk. Univer., Ser. Matematika, Mekhanika, Informatika, 2011, vol. 14, no. 27, pp. 46-60 (in Russian).

10. Danilin A.R., Kovrizhnykh O.O. Time-optimal control of a small mass point without environmental resistance. Dokl. Math., 2013, vol. 88, no. 1, pp. 465-467. doi: 10.1134/S1064562413040364.

11. Lee E.B., Markus L. Foundations of optimal control theory. N Y; London; Sydney: John Wiley \& Sons, Inc., 1967, 576 p. Translated to Russian under the title Osnovy teorii optimal'nogo upravleniya, Moscow: Nauka Publ., 1972, 576 p. ISBN: 0471522635.

12. Danilin A.R. Asymptotic behavior of the optimal cost functional for a rapidly stabilizing indirect control in the singular case. Comput. Math. Math. Physics, 2006, vol. 46, no. 12, pp. 2068-2079. doi: 10.1134/S0965542506120062 .

13. Il'in A.M., Danilin A.R. Asimptoticheskie metody $v$ analize [Asymptotic methods in analysis]. Moscow: Fizmatlit Publ., 2009, 248 p. ISBN: 978-5-9221-1056-3 .

14. Kantorovich L.V., Akilov G.P. Functional analysis. 2nd ed. N Y: Pergamon Press Ltd, 1982,589 p. ISBN: 0-08-023036-9. Original Russian text (3rd ed.) published in Kantorovich L.V., Akilov G.P. Funktsional'nyi analiz. Moscow: Nauka Publ., 1984, 752 p.

Received January 10, 2019

Revised February 4, 2019

Accepted February 11, 2019

Funding Agency: The second author was supported by the Russian Academic Excellence Project (agreement no. 02.A03.21.0006 of August 27, 2013, between the Ministry of Education and Science of the Russian Federation and Ural Federal University).

Aleksei Rufimovich Danilin, Dr. Phys.-Math. Sci., Prof., Krasovskii Institute of Mathematics and Mechanics of the Ural Branch of the Russian Academy of Sciences, Yekaterinburg, 620108 Russia; Ural Federal University, Yekaterinburg, 620002 Russia, e-mail: dar@imm.uran.ru .

Ol'ga Olegovna Kovrizhnykh, Cand. Sci. (Phys.-Math.), Krasovskii Institute of Mathematics and Mechanics of the Ural Branch of the Russian Academy of Sciences, Yekaterinburg, 620108 Russia; Ural Federal University, Yekaterinburg, 620002 Russia, e-mail: koo@imm.uran.ru .

Cite this article as: A. R. Danilin, O. O. Kovrizhnykh. Asymptotics of the solution to a singularly perturbed time-optimal control problem with two small parameters, Trudy Instituta Matematiki $i$ Mekhaniki URO RAN, 2019, vol. 25, no. 2, pp. 88-101. 